\title{
Acceptability, feasibility, and efficacy of Internet cognitive behavioral therapy (iCBT) for pediatric obsessive-compulsive disorder: a systematic review
}

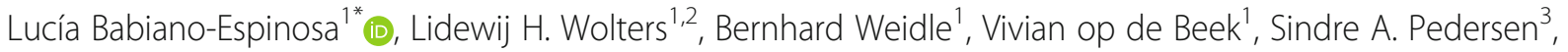 \\ Scott Compton ${ }^{4}$ and Norbert Skokauskas ${ }^{1}$
}

\begin{abstract}
Background: Obsessive-compulsive disorder (OCD) is a chronic mental health disorder characterized by recurring obsessions and compulsions affecting 1-3\% of children and adolescents. Current treatment options are limited by accessibility, availability, and quality of care. New technologies provide opportunities to address at least some of these challenges. This paper aims to investigate the acceptability, feasibility, and efficacy of traditional cognitive behavioral therapy with Internet cognitive behavioral therapy (iCBT) for pediatric OCD according to Preferred Reporting Items for Systematic Reviews and Meta-Analyses (PRISMA) guidelines.

Method: We searched EMBASE, Medline, PsycINFO, CENTRAL, LILACS, CINAHL, and Scopus. Results include articles from 1987 to March 2018. Main inclusion criteria were patients aged 4-18, primary diagnosis of OCD, and iCBT.

Results: Of the 2323 unique articles identified during the initial search, six studies with a total of 96 participants met our inclusion criteria: three randomized controlled trials, one single-case multiple-baseline design, one open-label trial, and one case series. Four studies reported a significant decrease in OCD severity on the Children's Yale-Brown Obsessive-Compulsive Scale (CY-BOCS) following iCBT, one study reported significant decrease in CY-BOCS scores for iCBT relative to waitlist, and the case series reported (some) symptom reduction in all participants. Six studies reported high rates of feasibility, and five studies reported good acceptability of iCBT.
\end{abstract}

Conclusion: At present, evidence regarding acceptability, feasibility, and efficacy of iCBT for pediatric OCD is limited. Results are promising but need to be confirmed and refined in further research.

Systematic review registration: PROSPERO CRD4201808587

Keywords: eHealth, Obsessive-compulsive disorder, Cognitive behavioral therapy, Adolescent, Child

\section{Background}

Obsessive-compulsive disorder (OCD) is a disabling mental health disorder affecting between 1 and $3 \%$ of children and adolescents [1]. OCD is characterized by disturbing recurring thoughts (obsessions) and repetitive behaviors (compulsions) [1] and is associated with significant impairment [2] and reduced quality of life [3].

\footnotetext{
* Correspondence: Lucia.babiano-espinosa@ntnu.no

'Department of Mental Health, Regional Centre for Child and Youth Mental Health and Child Welfare (RKBU), Norwegian University of Science and Technology (NTNU), Trondheim, Norway

Full list of author information is available at the end of the article
}

Without treatment, OCD has a chronic course in about $40-60 \%$ of those affected $[4,5]$.

Over the last three decades, OCD has moved from an almost untreatable, life-long psychiatric disorder to a highly manageable one. Two recent meta-analyses have supported cognitive behavioral therapy $(\mathrm{CBT})$ as the firstline treatment for children and adults with $\mathrm{OCD}[6,7]$ and two other meta-analyses reported larger effect sizes for CBT than for selective serotonin reuptake inhibitors (SSRIs) in pediatric OCD $[8,9]$. While relapse is common after cessation of medication, treatment gains from CBT appear more stable [10]. Still, CBT for pediatric OCD has

(c) The Author(s). 2019 Open Access This article is distributed under the terms of the Creative Commons Attribution 4.0 International License (http://creativecommons.org/licenses/by/4.0/), which permits unrestricted use, distribution, and reproduction in any medium, provided you give appropriate credit to the original author(s) and the source, provide a link to the Creative Commons license, and indicate if changes were made. The Creative Commons Public Domain Dedication waiver (http://creativecommons.org/publicdomain/zero/1.0/) applies to the data made available in this article, unless otherwise stated. 
not reached its full potential, with response rates ranging between 40 and $65 \%$ [11, 12]. In addition, stigma about mental health treatment in general and OCD in particular, limited access to high-quality CBT, and the high costs of CBT may reduce treatment uptake [13]. Sixty to $90 \%$ of adults with OCD from Western countries and China are not seeking treatment for OCD [14].

New technologies and increased access to the Internet provide unique opportunities to address some of these challenges by offering more interactive, childappealing [15], cost-effective [16], and more easily accessible therapies [17]. Illustrating this growing trend, the National Institute of Mental Health (NIMH) in the USA created the National Advisory Mental Health Council Workgroup on Opportunities and Challenges of Developing Information Technologies on Behavioral and Social Science Research [18]. Electronic and mobile health technologies are also included in the World Health Organization (WHO) Mental Health Action Plan 2013-2020 [19]. Internet cognitive behavioral therapy (iCBT) includes therapist-guided and automated interventions that are delivered using the Internet and information-technology based on cognitive behavioral therapy [20]. A recent systematic review indicated that Internet-based treatment programs for anxiety disorders and depression were generally well received by children and their parents [15]. These iCBT programs were effective in reducing anxiety symptoms, and some proved to be as effective as face-to-face interventions $[15,16,21]$. However, the effects on depression symptoms in adolescents and young adults (12-25 years) were small [16]. Previous meta-analysis has been published on iCBT for adult OCD showing good efficacy $[22,23]$. To our knowledge, no systematic review has investigated the acceptability, feasibility, and efficacy of Internet cognitive behavioral therapy (iCBT) for pediatric OCD. The present systematic review aims to bridge this gap.

\section{Method}

\section{Search strategy}

The first paper about OCD treatment involving computer technology was published in 1987 [24]. This systematic review included studies published from 1987 to March 2018. The Cochrane database was assessed to ensure that no similar systematic review had been published. We searched the seven relevant databases: EMBASE, Medline, PsyCINFO, CENTRAL, LILACS, CINAHL, and Scopus. The literature search involved a combination of thesaurus and free-text terms optimized to identify references containing three main concepts: "OCD," "Internet technology-based therapy," and "children or adolescents" Internet technology. (The exact keywords can be found in Additional file 2: study protocol.) V.B., S.P., and L.B.E. conducted the initial database research. L.W. and L.B.E independently filled the data collections forms that had been developed a priori by V.B. The data collection forms included (a) general information about the study (publication type, country of origin, funding), (b) study eligibility (inclusion criteria, sample details, study design, types of intervention, reasons to exclude), (c) study characteristics (aim, design, participants, outcomes), and (d) risk of bias assessment. L.W. and L.B.E. assessed article eligibility. In case of disagreement, consensus was reached through discussion with the other group members (B.W., N.S.). Relevant conference abstracts were searched manually to reduce potential limitations of the systematic database search. Finally, relevant Cochrane reviews, the WHO trials portal (ICTRP), ClinicalTrials.gov, and Google Scholar were searched to identify additional studies (see Additional file 2 for the study protocol).

\section{Inclusion and exclusion criteria}

Inclusion and exclusion criteria were based on the "PICOS" [25] approach to review empirical studies: population, intervention, comparators, outcomes, and study design.

\section{Inclusion criteria}

Population:

- Children and adolescents aged 4-18

- Primary diagnosis of obsessive-compulsive disorder diagnosed by a psychologist or psychiatrist according to DSM or ICD criteria

- All treatment settings

- Any cultural background, ethnicity, and sex

Intervention:

- CBT with Internet technology components

- No restrictions on therapist involvement or additional treatment

Comparator:

- Studies with and without comparators

- No restrictions were set on comparators.

Outcome:

- Treatment acceptability refers to the degree to which an individual perceives a treatment protocol as appropriate, fair, and reasonable for a given population or problem and any acceptability test is accepted as an outcome [26].

- Feasibility refers to whether treatment works in practice and drop-outs are accepted as main outcome [26]. 
- Treatment efficacy refers to the capacity to improving health-outcomes. Children's Yale-Brown Obsessive-Compulsive Scale (CY-BOCS) is accepted as the golden standard for its assessment [9].

For an overview of assessment instruments in this article, please see Additional file 3.

Study Design:

- Randomized controlled trial, blind trial, non-blind trial, adaptive clinical trial, non-randomized trial, interrupted time series design, cohort study, casecontrol study, and cross-sectional study published in English [27].

\section{Exclusion criteria}

Population:

- Adults

- Diagnosis of obsessive-compulsive disorder not determined by a qualified specialist (psychologist or psychiatrist) or not according to DSM or ICD criteria

Intervention:

- Other than CBT

\section{Comparator:}

- Studies with and without comparators are accepted.

- No restrictions were set on comparators.

Outcome:

- Not reporting on acceptability, feasibility, and efficacy.

Study Design:

- Qualitative study, commentary, correction, editorial letter (unless research letter reporting data), and single-case reports

\section{Results}

\section{Search results}

The initial search identified 3537 references. After removing 1214 duplicates, the search resulted in 2323 references. Of these, 2276 references were excluded after screening titles and abstracts, resulting in 47 references that were thoroughly screened. Forty-one from these 47 references were removed due to conflicts with selection criteria. Finally, six original studies were included in this systematic review (see Fig. 1 for flow diagram).
The reviewed studies included a total of 96 participants (47 girls, 49 boys) with a mean age ranging from 6.5 [28] to 14.4 years [29] (Table 1). Two studies included children aged $4-8$ years [28, 30], other studies included children and adolescents aged 7-17 years [29, 31, 32, 34]. The studies were conducted in North America [28, 30, 34], Australia [31], and Europe [29, 32]. There were three randomized controlled trials (RCTs) [29, 30, 34], one open trial [32], one single-case non-concurrent multiplebaseline design [31], and one case series [28] (Table 1). Two studies recruited families seeking treatment in outpatient clinics $[28,30]$. Three studies $[29,32,34]$ recruited participants from outpatient clinics and used advertisements in local newspapers, websites, and radio, and one study [31] combined advertisements in local newspapers with referrals from general practitioners.

Table 1 provides a description of the $\mathrm{iCBT}$ interventions. All studies included psychoeducation about OCD for the patients and their parents, as well as information about the treatment procedure. Exposure and response prevention (ERP) and cognitive interventions were key components in all procedures. All studies employed experienced clinicians [28-32, 34], and five studies also used psychology students as therapists [28-30, 32, 34]. Weekly supervisions for the therapists were performed to ensure the standards of treatment procedure [28-32, 34].

Two studies by Comer and colleagues and one by Farrell and colleagues provided a specific training for their therapists $[28,30,31]$. Therapist involvement varied from minimal (with occasional indirect contact via messages or phone) $[29,32]$ to substantial (via frequent video-teleconferencing) [34]. Parents were actively involved in the treatment process in all studies.

Two studies by Comer et al., and one by Farrell et al., provided a specific training for their therapists $[28,30,31]$. Therapist involvement varied from minimal (with occasional indirect contact via messages or phone) $[29,32]$ to substantial (via frequent videoteleconferencing) [34]. Parents were actively involved in the treatment process in all studies. Comer et al. first performed a case series [28] and subsequently an RCT [30] using the same iCBT concept. In the RCT, they compared 14 weeks face-to-face family-based CBT with family-based iCBT treatment. The iCBT included video-teleconferencing and interactive computer games that were added to enhance the children's understanding of the treatment concepts. Lenhard et al. [29, 32] evaluated "Internet Project for Children", an iCBT intervention delivered via an Internet platform with psychoeducational texts, films, animations, and exercises (12 sessions), in an open trial [32] followed by an RCT [29]. During this treatment, patients had irregular asynchronous contact with a therapist through messages and occasional telephone calls. 


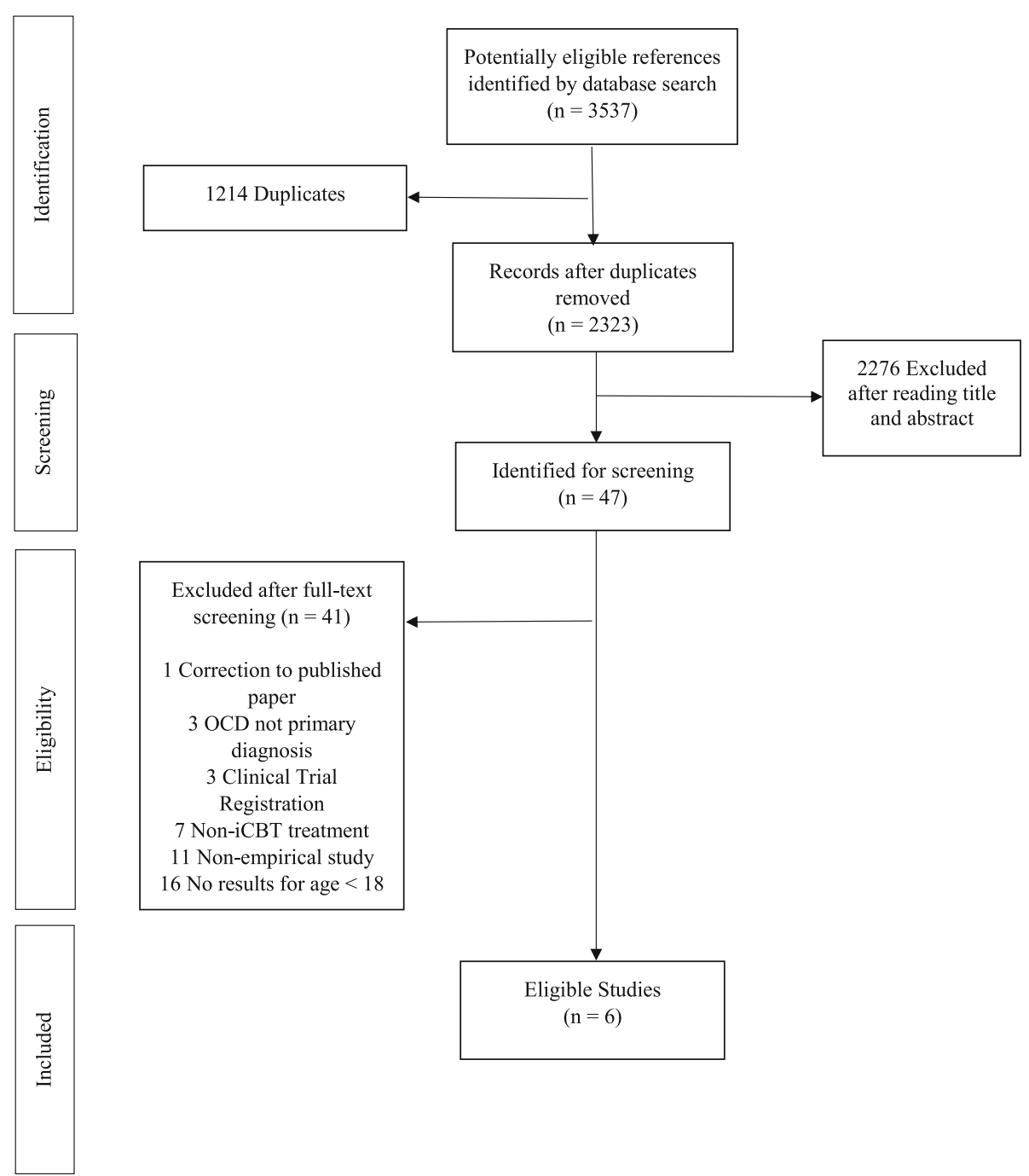

Fig. 1 Flowchart

Storch et al. [34] compared 14 CBT sessions delivered via video-teleconferencing with a waitlist control. Their iCBT program followed the Pediatric OCD Treatment Study (POTS) protocol with some adaptations, such as using email to send homework instructions [35]. Farrell et al. [31] evaluated a 6-week intensive treatment program combining $\mathrm{iCBT}$ and face-to-face CBT. This intervention included a 1-h face-to-face psychoeducation session and two face-toface intensive exposure and response prevention (ERP) sessions in 2 weeks, followed by a 3-week maintenance program delivered via video-teleconferencing.

\section{Acceptability}

Acceptability was examined using validated self-report questionnaires, such as the Client Satisfaction Questionnaire (CSQ) and the Working Alliance Inventory (WAI)
$[28,30]$, and several newly developed questionnaires (Tables 2 and 3) [29, 30, 32].

In the open trial by Lenhard et al., [32] treatment acceptability was evaluated in adolescents and at least one parent. The Internet Project for Children was rated as good or very good by the families [32]. In the following RCT, only adolescents' views were assessed [29]. Results showed that $46 \%$ of the adolescents were satisfied with the Internet Project for Children, $50 \%$ were satisfied most of the time but would have liked to meet a clinician occasionally (contact with a therapist was established through e-mail messages and phone calls only), and $4 \%$ would have preferred face-to-face treatment [29]. Other studies assessed parents' views only $[28,34]$. In both studies by Comer et al., all mothers [28, 30] reported good alliance with the therapist and that they were satisfied with the treatment. Storch et al. [34] reported very high satisfaction with treatment rated by parents. One study did not report on acceptability [31]. 


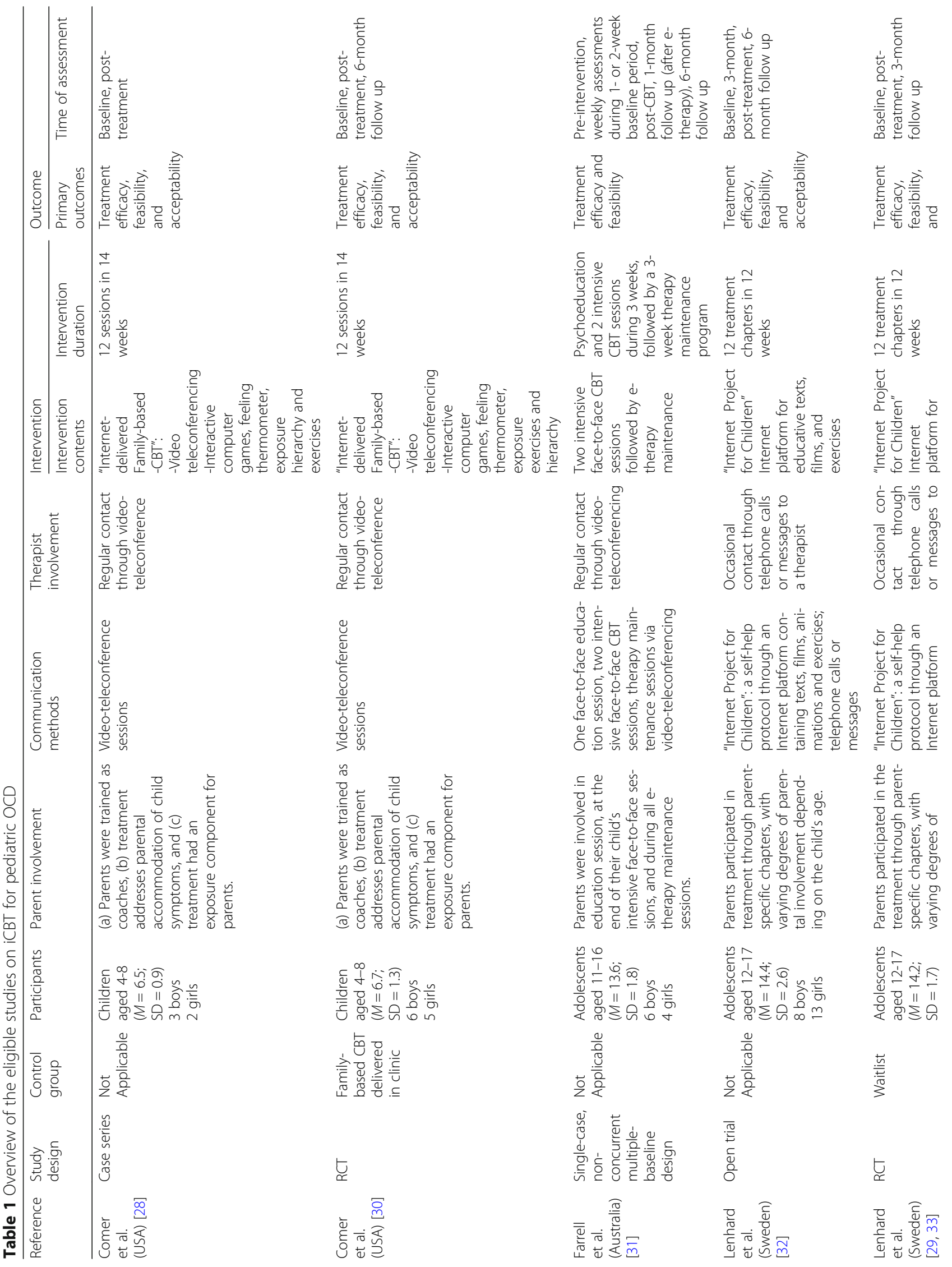




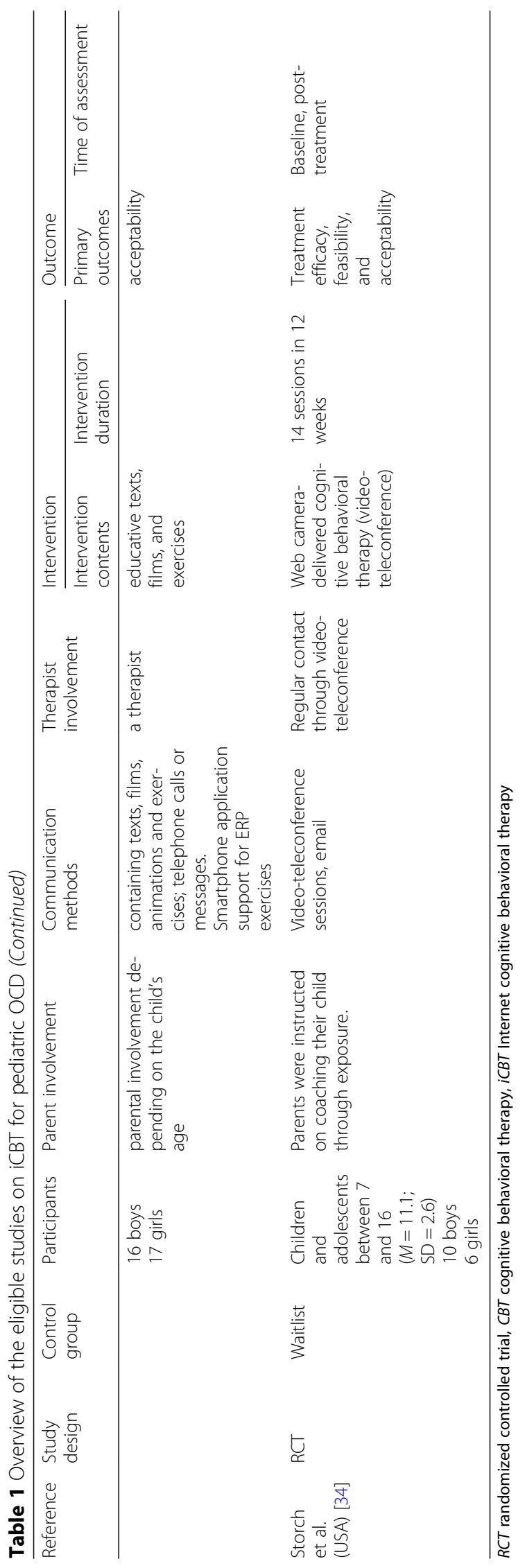




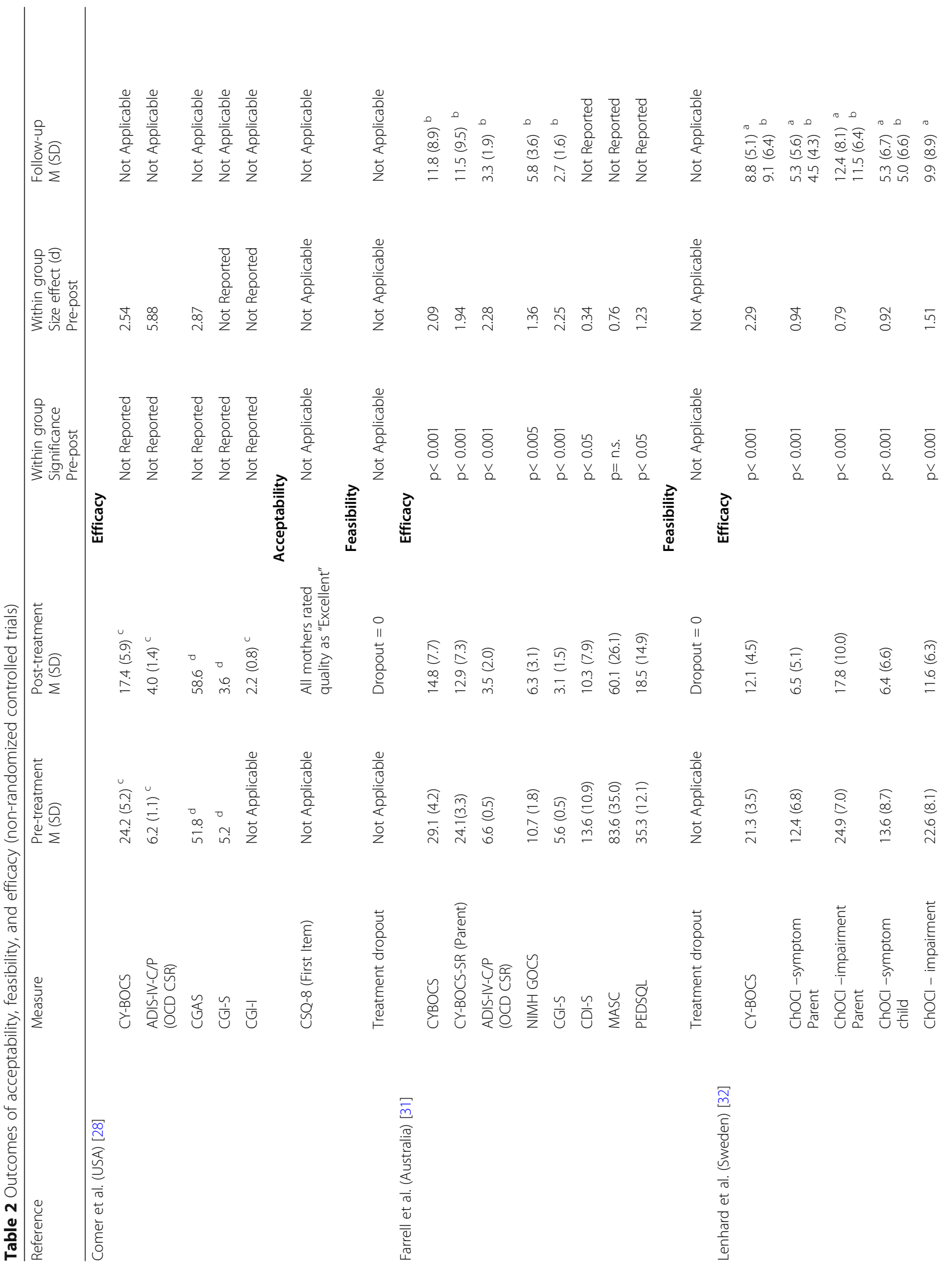




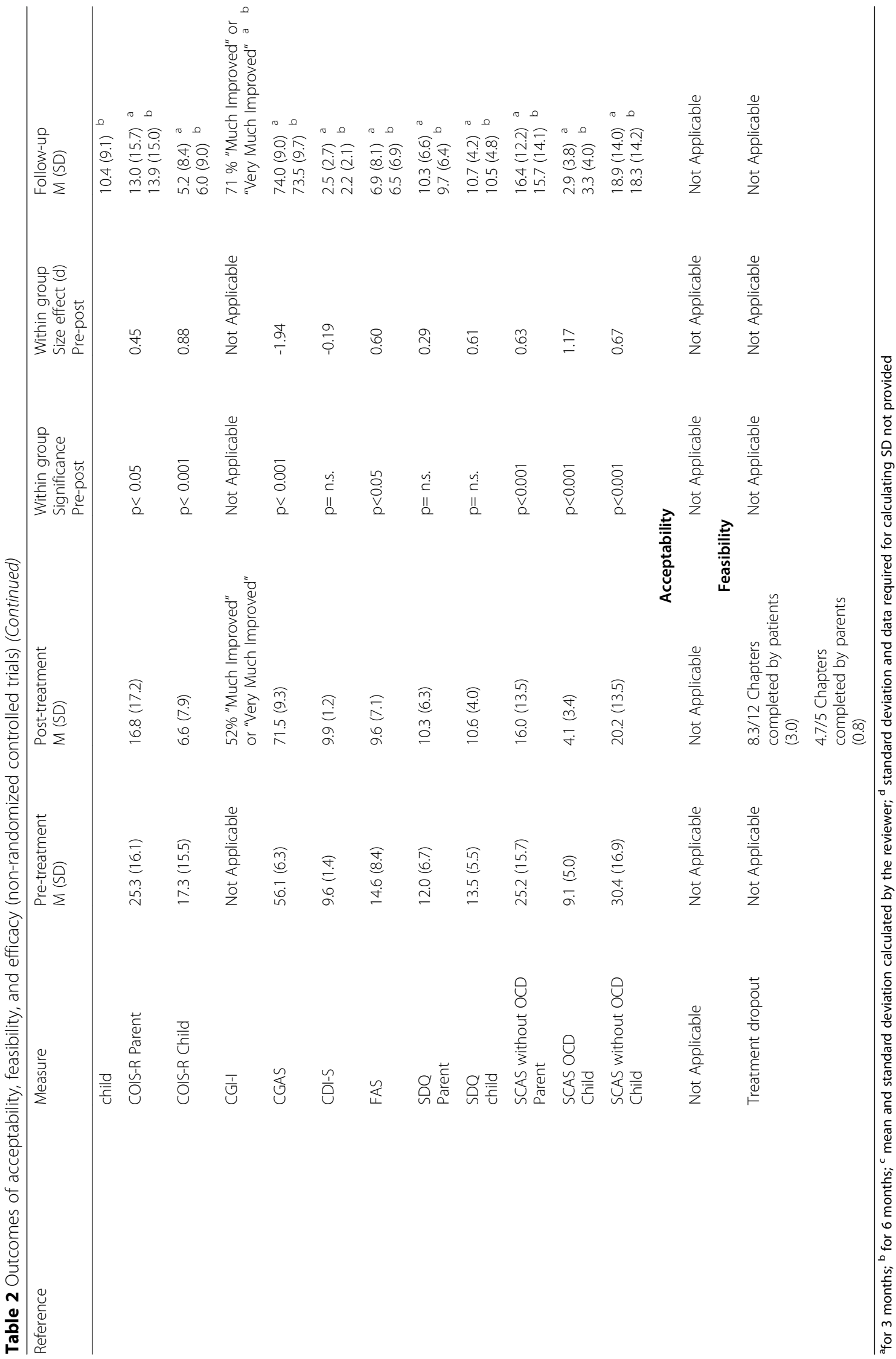




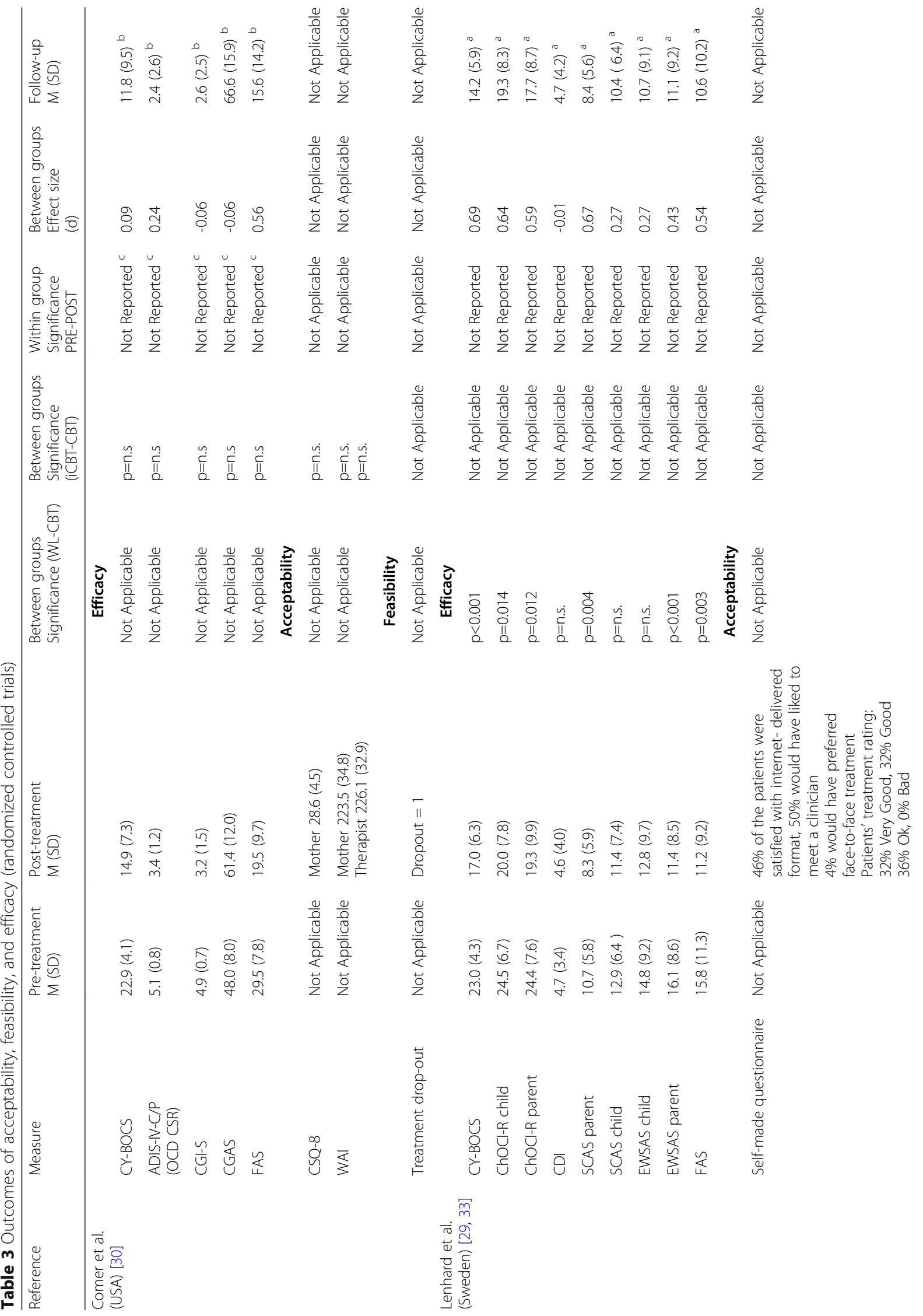




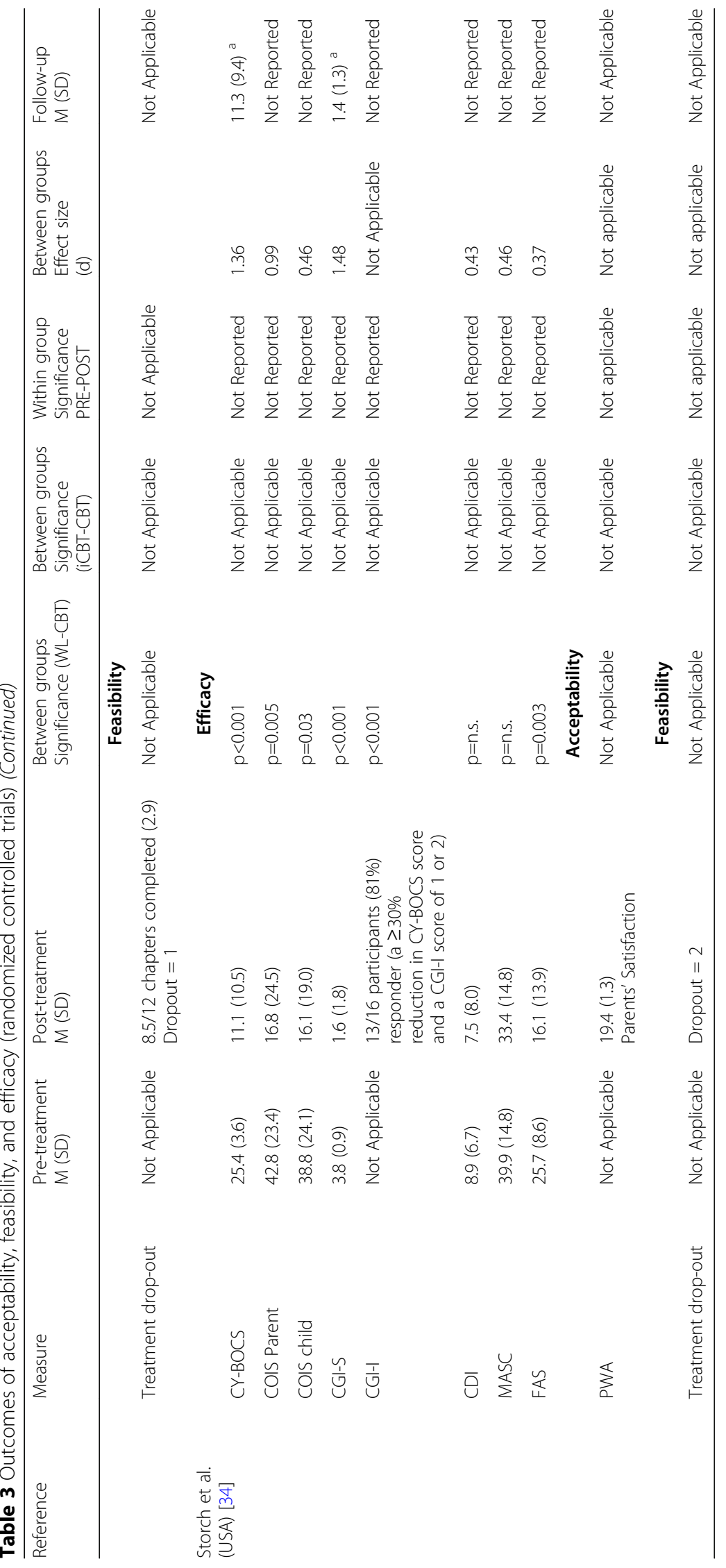




\section{Feasibility}

We examined treatment feasibility by documenting dropout from treatment, which ranged from none $[28,31]$ to two patients across all studies [34] (Tables 2 and 3). Altogether, $4.2 \%$ (four patients) dropped-out from all treatments. No participants dropped out from treatment in the case series study by Comer et al. [28], nor in the study by Farrell et al. [31]. One patient dropped out in the RCT by Comer et al. [30] (after session one; reason not reported). In Storch and colleagues' study [34], two participants withdrew from treatment due to a lack of perceived benefit. In the RCT from Lenhard et al. [29], one treatment drop-out was reported.

\section{Efficacy}

All studies used the Children's Yale-Brown ObsessiveCompulsive Scale (CY-BOCS) [36] to assess the severity of OCD symptoms (Tables 2 and 3). Four studies reported a statistically significant decrease in CY-BOCS scores from pre- to post-treatment [30-32, 34]. Comer et al. [28] reported that three of the five participants had a post-treatment CY-BOCS score $<16$ (clinical cut off), two other participants showed minimal improvement. Lenhard et al. reported a significant improvement in CY-BOCS score after iCBT [29]. Comer et al. [30] reported no significant difference between face-to-face CBT and iCBT.

Storch et al. [34] reported more than a half reduction $(56.1 \%)$ in OCD symptoms on the CY-BOCS following iCBT. Lenhard et al. [29, 32] reported an average reduction of $41 \%$ and $26 \%^{1}$ in CY-BOCS scores following iCBT in their open trial and RCT, respectively. Results from the study by Farrell et al. [31] showed $49 \%^{1}$ symptom reduction at post-treatment. Comer et al. reported a $35 \%^{1}$ and $28 \%^{1}$ average reduction in CY-BOCS scores at post-treatment in their RCT [30] and case series [28], respectively. In addition, four studies reported improvements at posttreatment on the Children's Global Assessment Scale (CGAS) [37] and the Clinical Global Impression Scale (CGI) [38] (Tables 2 and 3, see Additional file 3 for assessment glossary) [28, 30, 31, 34].

In their open trial, Lenhard et al. reported significant improvement at 3-month follow-up, which was maintained at 6-month follow-up [32]. In their RCT, participants continued to show significant improvement from post-treatment to 3-month follow-up [29]. Comer et al. reported significant improvement from pre-treatment to 6-month follow-up [30]. Farrell et al. reported 8 of 10 children in "reliable change" with at least 8.33 points in symptoms improvement on the

\footnotetext{
${ }^{1}$ Percentages based on group differences between mean pre- and mean post-treatment score.
}

CY-BOCS at post-treatment and 6-month follow-up (Tables 2 and 3) [31].

\section{Risk of bias}

As recommended by the Cochrane Collaboration, we used the Cochrane Collaboration's tool to assess risk of bias (low, unclear, or high-risk) among the eligible studies [39]. Overall results showed some risk of bias [39]. This was mainly due to the fact that even in the studies where a random generator was used to allocate participants to treatment condition, the need for the use of devices in the experimental condition (iCBT treatment), may be problematic for blinding participants to treatment condition [29, 30,34]. As a consequence of this, there may be an unclear risk bias in the blinding of outcomes category [28-32, 34] (Table 4).

\section{Discussion}

To our knowledge, this is the first systematic review on the acceptability, feasibility, and efficacy of iCBT for pediatric OCD. We identified six eligible studies involving four different $\mathrm{iCBT}$ interventions for pediatric OCD with a total number of 96 subjects.

The last decade has seen a substantial increase in emental health development and research [40]. The low number of eligible studies for pediatric OCD is in striking contrast to the rising use of Internet and mobile devices all over the world [41], their extensive use during childhood and adolescence [42], and the rising interest in iCBT [19]. There are more studies on $\mathrm{ICBT}$ in other populations than pediatric OCD. In 2015, a meta-analysis on iCBT for adult OCD included eight RCTs $(N=420)$ and reported no significant difference in efficacy between iCBT and face-to-face cognitive behavioral therapy [22]. In 2016, another meta-analysis included 18 studies and results showed large effect sizes for remote treatment for OCD in adults [23]. These results are in line with the results that we found in the present review. Furthermore, a recent meta-analysis on smartphone applications for depressive symptoms in adults [43] identified 18 RCTs assessing 22 smartphone applications, compared to only one smartphone application in our review [29]. Results of the present review showed that high treatment acceptability was reported in the five studies where acceptability was assessed [28-30, 32, 34]. However, different assessment tools were used (i.e., CSQ-8, PWA, WAI, self-developed questionnaires), and acceptability assessment tools were not always standardized or validated [29, 32]. Albeit opinions regarding treatment can differ considerably between respondents, some studies assessed only mothers' acceptance of the treatment $[28,30]$, some studies reported parents' acceptance (not specifying which parent) [32, 34], some studies assessed working alliance evaluated by the therapist [30], and only two studies assessed children's 


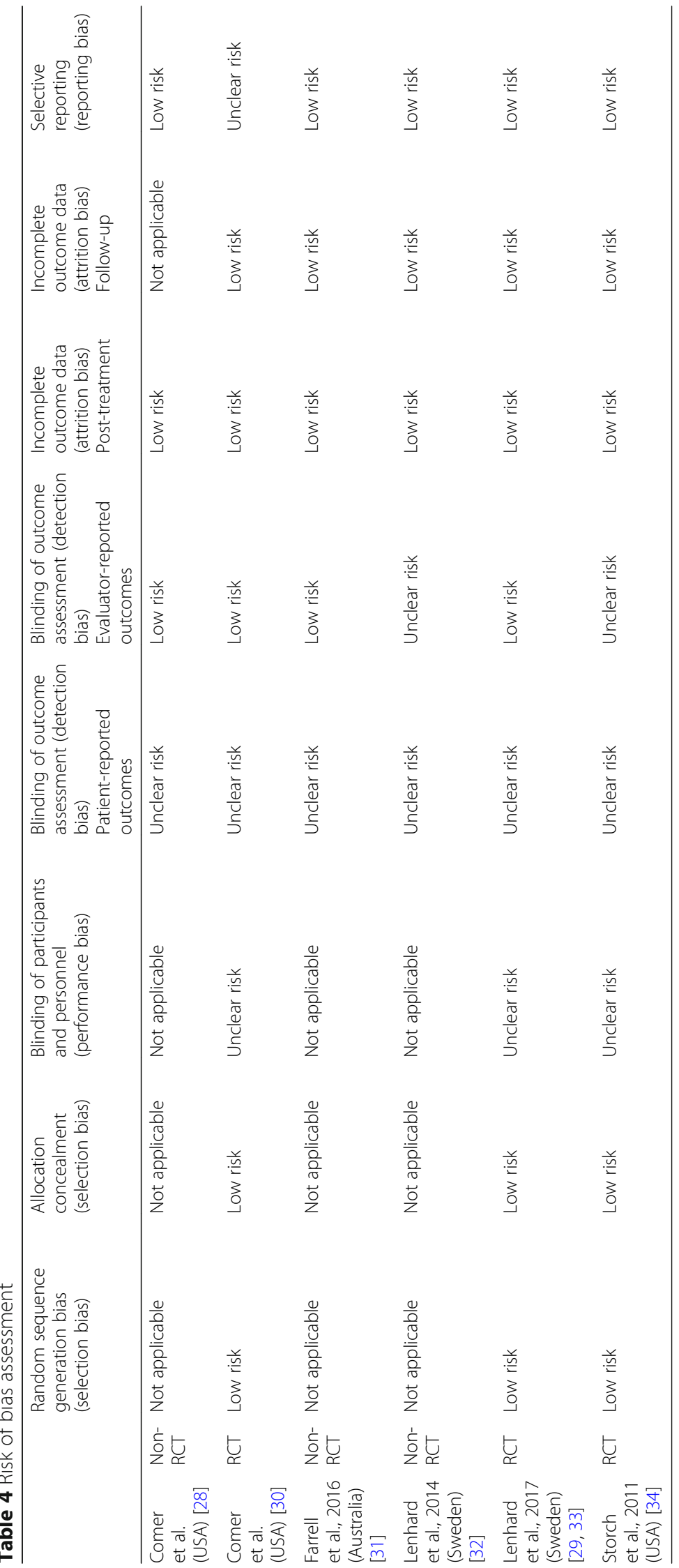


acceptance [29, 32]. Although acceptability was generally rated to be high in the study by Lenhard et al., [29] where therapist contact consisted of occassional e-mail, messages, and phone calls, half of participants reported that they would have liked to meet with a clinician occasionally, indicating that face-to-face therapist contact was an unmet need for part of this sample. In general, the findings in the present review regarding treatment acceptance are in line with the high acceptance of iCBT found for children with depression and anxiety [15]. Overall, thus far systematic reviews about Internet interventions for pediatric anxiety, depression, and internalizing problems have focused mainly on efficacy, and acceptability is generally under-reported [16, 21, 44].

Based on the low number of treatment drop-outs, ranging from none $[28,31,32]$ to two individuals [29, $30,34]$, feasibility was found to be high in all eligible studies. This is in line with two systematic reviews that reported good feasibility of Internet-assisted delivery of CBT for childhood anxiety and of web-based interventions for youth with internalizing problems $[15,44]$. However, these results should be interpreted with caution due to the small samples of the included studies.

All studies reported favorable effects of iCBT on OCD symptoms. The reported efficacy of iCBT in the reviewed studies ranged between $26 \%^{1}$ [29] and $56 \%{ }^{1}$ OCD symptom reduction [34]. A possible explanation for the variety in treatment effect is that development and application of iCBT programs are driven by different strategies. One strategy aims to overcome geographic barriers [34], other studies seek to improve limited response rates of conventionally delivered face-to-face CBT [28, 30, 31, 34], while another strategy aims to offer low-cost and easily accessible autonomous treatment programs [29, 32]. The heterogeneous results regarding efficacy should be interpreted according to the scope of the intervention.

Preliminary results indicate that treatment gains are maintained over time (3-6 months) [30-32]. While there is some evidence that treatment gains from face-to-face CBT on pediatric OCD are maintained at 1-year follow up [10], evidence concerning the sustainability of treatment results of iCBT is currently very limited.

None of the eligible studies reported a worsening of symptoms or any other treatment-related adverse events during iCBT. These results tentatively suggest that $\mathrm{iCBT}$ is a safe treatment. However, the spiraling growth of non-evidence-based e-health applications with poor guidance for users on how to make their choice causes concern [45]. Several potentially harmful effects, for example, regarding Internet security, confidentiality issues, and patient safety [46] were not assessed. This is a serious risk. A systematic review of Huckvale et al. [46] discovered systematic gaps for data security in $89 \%$ of the accredited health apps.

The main limitation of the current systematic review is the low number of eligible studies and their small samples [28-32, 34]. The six eligible studies came from three different continents (North America, Europe, and Australia) representing some cultural diversity although all belong to western cultures. As a result to our wide acceptance criteria, internal validity might be threatened [47]. There was a wide range of differences among the interventions, including the format of the intervention, the kind of Internet technology that was used, the length of treatment, and the amount of therapist contact. These differences make it difficult to draw an overall conclusion regarding the use of $\mathrm{iCBT}$ for pediatric OCD. However, the use of wide criteria made it possible to provide a complete overview of the state of the art in this field. In addition, wide inclusion criteria strengthened the external validity of this review, since the results show a realistic picture of the variety of $\mathrm{iCBT}$ treatments for pediatric OCD. A meta-analysis was not performed due to the small samples in the included studies, the low number of RCTs, and heterogeneity among treatments. Among the RCT's, two were superiority trials with waitlist as control group [29, 34], and one was a noninferiority trial with traditional face-to-face CBT as control group [30]. The two superiority trials $[29,34]$ examined very different iCBT treatments. One treatment consisted of a self-help program with minimal contact with the therapist [29]. The other treatment was based on regular contact with a therapist through videoteleconferencing [34]. These treatments aim to meet different needs for different patients. For these reasons, we believe that the results should be considered into the context of the treatments. Strengths of the present systematic review include the use of PRISMA guidelines to summarize and discuss the current state of acceptability, feasibility, and efficacy of iCBT for pediatric OCD (Additional file 1). These findings have importance for future directions. They do also raise questions requiring further research.

iCBT includes potential benefits offering CBT in a format that allows for reduced stigma and more widely available and accessible care. In addition, to meet the young patients in their area of expertise and using their "language" and way of cultural expression may enhance motivation for and adherence to the treatment program, which may contribute to more effective treatment and a reduced number of treatment drop-out. Studies exploring cost-effective and easy accessible autonomous treatment programs with minimal therapist contact are highly interesting in the scope of a stepped care model, allowing to differentiate between patients who benefit from this type of low-cost treatments and those who 
need therapist-delivered CBT [29, 32, 33]. In addition, intensive treatments, that could be delivered in varying formats, may be needed for other patients. A broader understanding about which format and amount of intervention works best for whom may lead to better outcomes and reduced societal costs. Future studies could focus on this question, assessing how different iCBT formats can augment traditional CBT by meeting the individual needs of patients. In addition, we need to know whether treatment gains obtained from these interventions will be maintained over time. It is also essential to assess and monitor potential adverse effects, for example, regarding Internet security, confidentiality issues, patient safety, and encryption. The use of smartphones, video-games, or health wearable trackers has the potential to both address barriers for treatment by adapting the therapy to the modern every-day life of the patient and to provide new possibilities for improved costeffectiveness. However, the currently available scientificevidence must improve substantially to enable the broader use of these new technologies.

\section{Conclusion}

Although e-mental health development and research have increased substantially over the last 10 years, the currently available evidence-base for iCBT programs for pediatric OCD is limited. The results in this systematic review indicate that $\mathrm{iCBT}$ can be a feasible and acceptable treatment. Available limited evidence supports the use of i-tools to enhance ERP exercises and overcome barriers to treatment. However, replication studies with bigger samples are needed, along with studies testing which modalities and components for iCBT are most effective for whom.

\section{Supplementary information}

Supplementary information accompanies this paper at https://doi.org/10. 1186/s13643-019-1166-6.

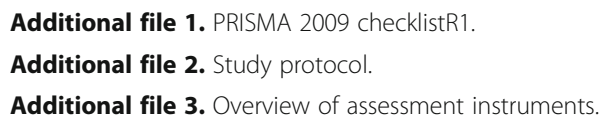

\section{Abbreviations}

CBT: Cognitive behavioral therapy; CENTRAL: The Cochrane Central Register of Controlled Trials; CGAS: Children's Global Assessment Scale; CGI: Clinical Global Impression Scale; CINAHL: Cumulative Index of Nursing and Allied Health Literature; CSQ-8: The Client Satisfaction Questionnaire-8; CYBOCS: Children's Yale-Brown Obsessive-Compulsive Scale; DSM: The Diagnostic and Statistical Manual of Mental Disorders; EMBASE: Excerpta Medica dataBASE; ERP: Exposure and response prevention; iCBT: Internet cognitive behavioral therapy; ICD: International Statistical Classification of Diseases and Related Health Problems; ICTRP: International Clinical Trials Registry Platform; LILACS: Latin American and Caribbean Health Sciences Literature; NIMH: National Institute of Mental Health; OCD: Obsessive Compulsive Disorder; PICOS: Population, intervention, comparators, outcomes, and study design; PRISMA: Preferred Reporting Items for Systematic Reviews and Meta-Analyses; PWA: Parent Working Alliance;
RCT: Randomized controlled trial; SSRIs: Selective serotonin reuptake inhibitors; WAl: Working Alliance Inventory; WHO: World Health Organization

\section{Acknowledgements}

No acknowledgements

\section{Authors' contributions}

LBE contributed to the acquisition, analysis, and interpretation of data for the work (assessing independently the identified trials to determine eligibility, risk of bias assessment) and drafting of the work. LW contributed to the conception and design of the work and the acquisition, analysis, and interpretation of data for the work (assessing independently the identified trials to determine eligibility and risk of bias assessment), revising the work critically for important intellectual content. BW contributed to the design of the work, revising the work critically for important intellectual content. VB contributed to the conception and design of the work (protocol drafting) and the acquisition, analysis, interpretation of data for the work (preliminar searches), revising the work critically for important intellectual content. SP contributed to the acquisition and analysis (database searches), revising the work critically for important intellectual content. SC contributed to the design of the work, revising the work critically for important intellectual content. NS contributed to the conception and design of the work and the acquisition, analysis, and interpretation of data for the work (assessing independently the identified trials to determine eligibility and risk of bias assessment), revising the work critically for important intellectual content. All authors agree both to be personally accountable for the author's own contributions and to ensure that questions related to the accuracy or integrity of any part of the work, even ones in which the author was not personally involved, are appropriately investigated, resolved, and the resolution documented in the literature. All authors approved of the final version to be published.

\section{Funding}

The Liaison Committee for Education, Research and Innovation in Central Norway supported this work (Samarbeidsorganet mellom Helse Midt-Norge RHF og NTNU).

\section{Availability of data and materials}

All papers available online: systematic review

Ethics approval and consent to participate

Not applicable: systematic review

Consent for publication

Not applicable: systematic review

\section{Competing interests}

The authors declare that they have no competing interests.

\section{Author details}

'Department of Mental Health, Regional Centre for Child and Youth Mental Health and Child Welfare (RKBU), Norwegian University of Science and Technology (NTNU), Trondheim, Norway. ${ }^{2}$ De Bascule, Academic Center for Child and Adolescent Psychiatry, Center of Expertise for OCD, Anxiety and Tics, Meibergdreef 5, 1105, AZ, Amsterdam, The Netherlands. ${ }^{3}$ Health, Library Section for Medicine and Health Sciences, Norwegian University of Science and Technology, Olav Kyrres gate 9, 7030 Trondheim, Norway. ${ }^{4}$ Department of Psychiatry and Behavioral Sciences, Duke University School of Medicine, 2200 W Main St \#340, Durham, NC 27705, USA.

Received: 20 March 2019 Accepted: 21 September 2019

Published online: 20 November 2019

References

1. American Psychiatric Association. Diagnostic and statistical manual of mental disorders. Arlington: Am Psychiatric Assoc; 2013.

2. Piacentini J, Bergman RL, Keller M, McCracken J. Functional impairment in children and adolescents with obsessive-compulsive disorder. J Child Adolesc Psychopharmacol. 2003;13(Suppl 1):S61-9. 
3. Weidle B, Ivarsson T, Thomsen PH, Lydersen S, Jozefiak T. Quality of life in children with OCD before and after treatment. Eur Child Adolesc Psychiatry. 2015;24(9):1061-74.

4. Skoog G, Skoog I. A 40-year follow-up of patients with obsessivecompulsive disorder. Arch Gen Psychiatry. 1999;56(2):121-7.

5. Stewart SE, McKenney KS, Simpson A, Belschner L, Lin S. Group and family cognitive behavioral therapy for pediatric obsessive-compulsive disorder: Examining traditional and novel outcomes. J Am Acad Child Adolesc Psychiatry. 2016;55(10 Supplement 1):S341.

6. Olatunji BO, Davis ML, Powers MB, Smits JA. Cognitive-behavioral therapy for obsessive-compulsive disorder: a meta-analysis of treatment outcome and moderators. J Psychiatr Res. 2013;47(1):33-41.

7. Wu Y, Lang Z, Zhang H. Efficacy of cognitive-behavioral therapy in pediatric obsessive-compulsive disorder: a meta-analysis. Med Sci Monit. 2016;22:1646-53.

8. Ivarsson T, Skarphedinsson G, Kornør H, Axelsdottir B, Biedilae S, Heyman I, et al. The place of and evidence for serotonin reuptake inhibitors (SRIs) for obsessive compulsive disorder (OCD) in children and adolescents: views based on a systematic review and meta-analysis. Psychiatry Res. 2015;227(1):93-103.

9. McGuire JF, Piacentini J, Lewin AB, Brennan EA, Murphy TK, Storch EA. A meta-analysis of cognitive behavior therapy and medication for child obsessive-compulsive disorder: moderators of treatment efficacy, response, and remission. Depress Anxiety. 2015;32(8):580-93.

10. Højgaard DRMA, Hybel KA, Ivarsson T, Skarphedinsson G, Becker Nissen J, Weidle B, et al. One-year outcome for responders of cognitive-behavioral therapy for pediatric obsessive-compulsive disorder. J Am Acad Child Adolesc Psychiatry. 2017;56(11):940-7.e1.

11. Torp NC, Dahl K, Skarphedinsson G, Thomsen PH, Valderhaug R, Weidle B, et al. Effectiveness of cognitive behavior treatment for pediatric obsessive-compulsive disorder: acute outcomes from the Nordic Long-term OCD Treatment Study (NordLOTS). Behav Res Ther. 2015;64:15-23.

12. Watson HJ, Rees CS. Meta-analysis of randomized, controlled treatment trials for pediatric obsessive-compulsive disorder. J Child Psychol Psychiatry. 2008; 49(5):489-98.

13. Wolters LH, op de Beek V, Weidle B, Skokauskas N. How can technology enhance cognitive behavioral therapy: the case of pediatric obsessive compulsive disorder. BMC Psychiatry. 2017;17(1):226.

14. Schwartz C, Schlegl S, Kuelz AK, Voderholzer U. Treatment-seeking in OCD community cases and psychological treatment actually provided to treatment-seeking patients: a systematic review. J Obsessive Compulsive Relat Disord. 2013;2(4):448-56.

15. Rooksby M, Elouafkaoui P, Humphris G, Clarkson J, Freeman R. Internet-assisted delivery of cognitive behavioural therapy (CBT) for childhood anxiety: systematic review and meta-analysis. J Anxiety Disord. 2015;29:83-92.

16. Pennant ME, Loucas CE, Whittington C, Creswell C, Fonagy P, Fuggle P, et al. Computerised therapies for anxiety and depression in children and young people: a systematic review and meta-analysis. Behav Res Ther. 2015; 67:1-18.

17. Newman MG, Szkodny LE, Llera SJ, Przeworski A. A review of technologyassisted self-help and minimal contact therapies for anxiety and depression: is human contact necessary for therapeutic efficacy? Clin Psychol Rev. 2011; 31(1):89-103.

18. National Institute of Mental Health. Opportunities and challenges of developing information technologies on behavioral and social science clinical research: National Institute of Mental Health. 2017. Available from: https://www.nimh.nih.gov/about/advisory-boards-and-groups/namhc/ reports/opportunities-and-challenges-of-developing-informationtechnologies-on-behavioral-and-social-science-clinical-research.shtml

19. WHO. Mental health action plan 2013-2020. 2013 [cited 2018 2018-10-25]. Printed by the WHO document production services. Available from: http:// apps.who.int/iris/bitstream/handle/10665/89966/9789241506021_eng.pdf; jsessionid=18D3E9EAAC4ECC5E2577A81623BDCDE1 ? sequence $=1$.

20. Andersson G, Carlbring P, Lindefors N. History and Current Status of ICBT. In: Lindefors N., Andersson G. (eds). Guided Internet-Based Treatments in Psychiatry. Cham; Springer: 2016. https://doi.org/10.1007/978-3-319-06083$5+1$

21. Ye X, Bapuji SB, Winters SE, Struthers A, Raynard M, Metge C, et al. Effectiveness of internet-based interventions for children, youth, and young adults with anxiety and/or depression: a systematic review and metaanalysis. BMC Health Serv Res. 2014;14(1):313.

22. Dettore D, Pozza A, Andersson G. Efficacy of technology-delivered cognitive behavioural therapy for OCD versus control conditions, and in comparison with therapist-administered CBT: meta-analysis of randomized controlled trials. Cogn Behav Ther. 2015;44(3):190-211.

23. Wootton BM. Remote cognitive-behavior therapy for obsessive-compulsive symptoms: a meta-analysis. Clin Psychol Rev. 2016;43:103-13.

24. Baer $L$, Minichiello WE, Jenike MA. Use of a portable-computer program in behavioral treatment of obsessive-compulsive disorder. Am J Psychiatry. 1987;19:237-40

25. Beller EM, Glasziou PP, Altman DG, Hopewell S, Bastian H, Chalmers I, et al. PRISMA for abstracts: reporting systematic reviews in journal and conference abstracts. PLoS Med. 2013;10(4):e1001419.

26. Myers KM, Valentine JM, Melzer SM. Feasibility, acceptability, and sustainability of telepsychiatry for children and adolescents. Psychiatr Serv. 2007;58(11):1493-6.

27. Morrison A, Polisena J, Husereau D, Moulton K, Clark M, Fiander M, et al. The effect of English-language restriction on systematic review-based metaanalyses: a systematic review of empirical studies. Int J Technol Assess Health Care. 2012;28(2):138-44.

28. Comer JS, Furr JM, Cooper-Vince CE, Kerns CE, Chan PT, Edson AL, et al. Internet-delivered, family-based treatment for early-onset OCD: a preliminary case series. J Clin Child Adolesc Psychol. 2014;43(1):74-87.

29. Lenhard F, Anderson E, Mataix-Cols D, Rück C, Vigerland S, Högström J, Hillborg M, et al. Therapist-guided, Internet-delivered cognitive-behavioral therapy for adolescents with obsessive-compulsive disorder: a randomized controlled trial. J Am Acad Child Adolesc Psychiatry. 2017;56(1):10-19.e2.

30. Comer JS, Furr JM, Kerns CE, Miguel E, Coxe S, Meredith Elkins R, et al. Internet-delivered, family-based treatment for early-onset OCD: a pilot randomized trial. J Consult Clin Psychol. 2017;85(2):178-86.

31. Farrell LJ, Oar EL, Waters AM, McConnell H, Tiralongo E, Garbharran V, et al. Brief intensive CBT for pediatric OCD with E-therapy maintenance. J Anxiety Disord. 2016:42:85-94

32. Lenhard F, Vigerland S, Andersson E, Ruck C, Mataix-Cols D, Thulin U, et al. Internet-delivered cognitive behavior therapy for adolescents with obsessive-compulsive disorder: an open trial. PLoS One. 2014;9(6):e100773.

33. Lenhard F, Ssegonja R, Andersson E, Feldman I, Ruck C, Mataix-Cols D, et al. Cost-effectiveness of therapist-guided internet-delivered cognitive behaviour therapy for paediatric obsessive-compulsive disorder: results from a randomised controlled trial. BMJ Open. 2017;7(5):e015246.

34. Storch EA, Caporino NE, Morgan JR, Lewin AB, Rojas A, Brauer L, et al. Preliminary investigation of web-camera delivered cognitive-behavioral therapy for youth with obsessive-compulsive disorder. Psychiatry Res. 2011; 189(3):407-12.

35. Pediatric O. Cognitive-behavior therapy, sertraline, and their combination for children and adolescents with obsessive-compulsive disorder: the Pediatric OCD Treatment Study (POTS) randomized controlled trial. JAMA. 2004; 292(16):1969.

36. Goodman WK, Price LH. Assessment of severity and change in obsessive compulsive disorder. Psychiatr Clin North Am. 1992;15(4):861-9.

37. Jozefiak T, Hanssen-Bauer K, Bjelland I. Psychometric properties of the Norwegian version of Children's Global Assessment Scale (CGAS). PsykTestBarn. 2018;1:3.

38. Busner J, Targum SD. The clinical global impressions scale: applying a research tool in clinical practice. Psychiatry (Edgmont). 2007:4(7):28.

39. Higgins JP, Altman DG, Gøtzsche PC, Jüni $P$, Moher D, Oxman AD, et al. The Cochrane Collaboration's tool for assessing risk of bias in randomised trials. BMJ. 2011;343:d5928.

40. Firth J, Torous J, Yung AR. Ecological momentary assessment and beyond: the rising interest in e-mental health research. J Psychiatr Res. 2016;80:3-4

41. The World Bank. World development report 2016: Digital dividends. 2016 [Available from: http://www.worldbank.org/en/publication/wdr2016.

42. Ólafsson K. Livingstone S, Haddon L. Children's use of online technologies in Europe: a review of the European evidence base. 2013 [Available from: http://www.Ise.ac.uk/media@|se/research/EUKidsOnline/EU\%20Kids\%20III/ Reports/D2.2ReviewEvidenceDatabase.pdf.

43. Firth J, Torous J, Nicholas J, Carney R, Pratap A, Rosenbaum S, et al. The efficacy of smartphone-based mental health interventions for depressive symptoms: a meta-analysis of randomized controlled trials. World Psychiatry. 2017;16(3):287-98

44. Reyes-Portillo JA, Mufson L, Greenhill LL, Gould MS, Fisher PW, Tarlow N, et al. Web-based interventions for youth internalizing problems: a systematic review. J Am Acad Child Adolesc Psychiatry. 2014;53(12):254-1270.e5.

45. Anthes E. Pocket psychiatry. Nature. 2016;532(7597):20-3. 
46. Huckvale K, Prieto JT, Tilney M, Benghozi P-J, Car J. Unaddressed privacy risks in accredited health and wellness apps: a cross-sectional systematic assessment. BMC Med. 2015;13(1):214.

47. Rothwell PM. External validity of randomised controlled trials:"to whom do the results of this trial apply?". Lancet. 2005;365(9453):82-93.

\section{Publisher's Note}

Springer Nature remains neutral with regard to jurisdictional claims in published maps and institutional affiliations.

Ready to submit your research? Choose BMC and benefit from:

- fast, convenient online submission

- thorough peer review by experienced researchers in your field

- rapid publication on acceptance

- support for research data, including large and complex data types

- gold Open Access which fosters wider collaboration and increased citations

- maximum visibility for your research: over $100 \mathrm{M}$ website views per year

At $\mathrm{BMC}$, research is always in progress.

Learn more biomedcentral.com/submissions 